\section{A 5,500-Year Model of Changing Crop Niches on the Tibetan Plateau}

\section{Jade d'Alpoim Guedes, Sturt W. Manning, and R. Kyle Bocinsky}

Department of Anthropology, Washington State University, Pullman, Washington 99163, USA (jade.dalpoimguedes@wsu .edu, bocinsky@wsu.edu) (d’Alpoim Guedes and Bocinsky)/ Cornell Tree-Ring Laboratory, Cornell University, Ithaca, New York, 14853, USA (Manning)/Crow Canyon Archaeological Center, Cortez, Colorado 81321, USA (Bocinsky). This paper was submitted 9 VIII 15, accepted 22 XII 15, and electronically published 13 VII 16.

CA+ Online-Only Material: Supplement A, data file, video file

The timing and mechanics of the spread of agriculture to the Tibetan Plateau - one of the most challenging environmental contexts on earth - is a focus of recent work and debate. Understanding the timing and spread of agriculture is basic to archaeology and history worldwide. Researchers seek evidence for the earliest, furthest, or highest occurrences of diagnostic elements. However, the Tibetan Plateau case illustrates a key flaw in current work: archaeologists have often uncritically interpreted the presence of plant domesticates at archaeological sites as being indicative of local agricultural practice. This assumption neglects the long history of food exchange on the Plateau, as elsewhere in the world, even beyond what were then the limits of agriculture. The cause is a fundamental lack of understanding of where crops could be grown in prehistory. Using a formal model of the agricultural thermal niche between the $5500 \mathrm{BP}$ and the present, we argue that agricultural niches on the Tibetan Plateau were tightly constrained to lower-elevation river valleys throughout time. This pattern is confirmed by analysis of the extent of modern crop production on the Plateau. The challenges deriving from these altitudinal constraints placed on early Tibetans largely explain how and why the Tibetan economy developed in the way that it did.

In archaeology, our understanding of how changes in climate impacted human economies has been limited by our failure to identify the mechanisms via which these changes impact the basis of human economies. The Tibetan Plateau is an area of the world that experienced a major transition in farming systems. Recent data from the southeastern and northeastern Tibetan Plateau (SETP and NETP, respectively; fig. 1) have shown that, following two millennia of broomcorn and foxtail

(C) 2016 by The Wenner-Gren Foundation for Anthropological Research. All rights reserved. 0011-3204/2016/5704-0008\$10.00. DOI: 10.1086 1687255 millet cultivation in the margins of the Tibetan Plateau, these crops were largely abandoned in favor of two newly introduced domesticates: wheat and barley (Chen et al. 2015; d'Alpoim Guedes 2013, 2015; d'Alpoim Guedes, Bocinsky, and Butler 2015; d'Alpoim Guedes and Butler 2014; d'Alpoim Guedes et al. 2015; Dong et al. 2015). However, to date, explanatory frameworks have only rarely used models to predict how these crops were impacted by changes in climate over the course of the Holocene (d'Alpoim Guedes 2013; d'Alpoim Guedes, Bocinsky, and Butler 2015; d'Alpoim Guedes and Butler 2014; d'Alpoim Guedes et al. 2015). Until recently, archaeologists have not been able to accurately reconstruct when and where ancient crops could be cultivated. Yet when and where these crops could have been grown in the past has profound implications for our interpretation of the archaeological record (d'Alpoim Guedes, Bocinsky, and Butler 2015). Ethnographic and archaeological studies on the spread of agricultural products and technology across the world have revealed that complex interactions took place between huntergatherer and farmer populations (d'Alpoim Guedes 2015). Agriculturalists are constrained by the effective altitudinal limits of the crops they cultivated (given contemporary climate parameters). To reveal these interactions in the archaeological record, we need to have a good understanding of the areas farmers, foragers, and pastoralists were able to occupy in the past.

We describe the creation of an agroecological thermal niche model (d'Alpoim Guedes 2013; d'Alpoim Guedes and Butler 2014; Bocinsky and Kohler 2014) of wheat, barley, broomcorn, and foxtail millet from $5500 \mathrm{BP}$ to the present day that allows archaeologists to predict the area crops could occupy in the past. This model demonstrates, contrary to assertions made by earlier studies (Chen et al. 2015; Dong et al. 2015), that there is no evidence Tibetan farmers were able to push the limits of barley cultivation above 4,000 m asl. Rather, Tibetan farmers in the past, as today, practiced agriculture in the deeply cut lower-altitude river valleys on the Tibetan Plateau. This has profound implications for the manner in which agriculture spread to this environment. We argue that, contrary to what was the case in flatter areas of the world, agriculturalists were not able to spread into high-altitude environments as a "wave of advance." Rather, agriculturalists were likely tightly constrained in select river valleys, leaving large parts of the Plateau as potential refugia for hunter-gatherers. In later periods of time, pastoral animals allowed humans to carry stored foodstuffs across larger areas of the Plateau and so enabled the formation of the traditional Tibetan "samadrok" economy (d'Alpoim Guedes 2015; Kapstein 2006). However, agricultural activity remained constrained, as it always has been, to these lowerlying valleys. An analysis of the elevation of modern cropland on the Tibetan Plateau (above 1,500 m asl; fig. A1; figs. A1-A5 available in $\mathrm{CA}+$ online supplement A.) confirms these modeled constraints-showing that $95 \%$ of cropland is at elevations 


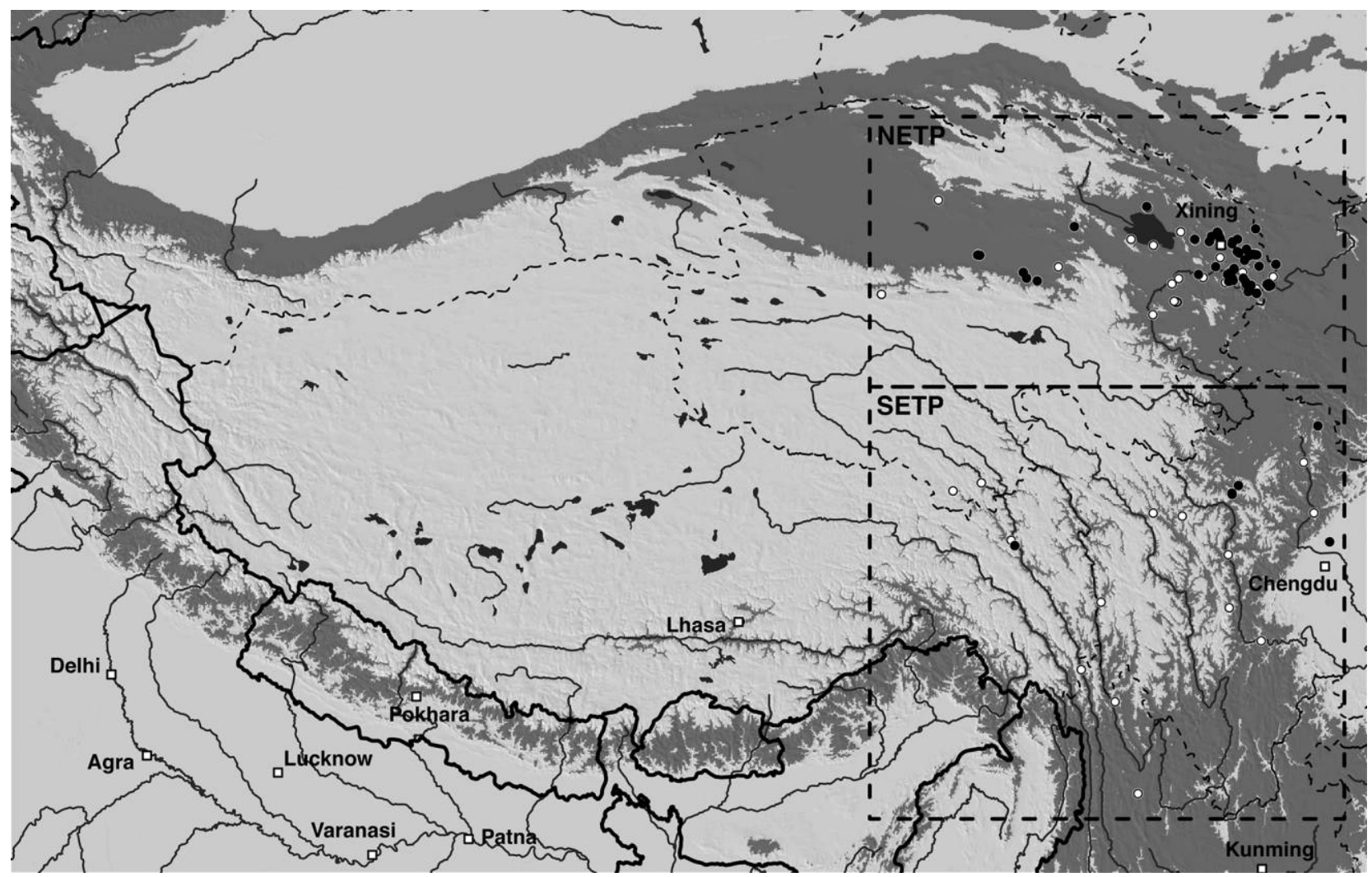

Figure 1. The Tibetan Plateau. The dark gray area indicates elevations between 1,500 and 4,000 $\mathrm{m}$ asl and defines the region analyzed in this study. The northeastern Tibetan Plateau (NETP) and southeastern Tibetan Plateau (SETP) regions are shown in dashed boxes. Archaeological sites are shown as dots; black dots are sites with ${ }^{14} \mathrm{C}$-dated materials; white dots are recorded sites that have not been ${ }^{14} \mathrm{C}$ dated. Many sites are clustered, and so coloration of the dots might be obscured. Refer to table A1 for sites included in our radiocarbon database. A color version of this figure is available online.

below $3,314 \mathrm{~m}$ asl and $99.98 \%$ of cropland exists below $4,000 \mathrm{~m}$ asl (fig. A2).

Our thermal niche model reveals that warmer temperatures between 5500-4200 BP permitted millets to be grown across most of the areas below $3,000 \mathrm{~m}$ asl on the NETP and SETP (fig. 2); these areas have a high (>95\%) probability of being in the niche in any given year. Between 3750 and $3010 \mathrm{BP}$, the probability that these areas were in the niche decreases to $80 \%$ 90\% (fig. 2 and video A1, available in CA+ supplement A). Millets appear to lose their range in these river valleys by around $3000 \mathrm{BP}$, with the probability of these lower-altitude areas being in the niche decreasing to $60 \%$. During this period of time, only valleys between 2,000 and $2,500 \mathrm{~m}$ asl have a probability of being in the niche of $70 \%$ or higher. It is worth noting, however, that there is a wide standard deviation in the reconstruction of millet's niche due to the paucity of experimental data on these crops. In reality, it is likely that millets may require more growing degree days (GDDs) to support their growth cycle than those that we report here (and, consequently, that their niche would be more restrictive). This pattern is reinforced by a summed probability distribution of available ra- diocarbon dates from across the Plateau representing human activity (fig. 4). In both the NETP and the SETP, millets fall out of the record between 4000 and 3500 BP (fig. 4). This is a period that corresponds to a decrease in temperature in Marcott et al. (2013), when the probability of being in the millet niche across the Plateau was roughly $70 \%$ (fig. 3 ); there is also a concurrent sharp reduction in East Asian summer monsoon (EASM) intensity and a change (inflection) in temperature and EASM trend trajectories (fig. A3), likely associated with changes in intertropical convergence zone position and hence El Niño southern oscillation variability (Dykoski et al. 2005:82-83). Observed (versus climate model simulated) data indicate just such a general association over longer timescales (e.g., centennial) of cooler centuries with drier conditions in monsoonal Asia (Rehfeld and Laepple 2016). All these phenomena are perhaps associated (in the long term) with orbital forcing changes in summer/winter insolation (Berger and Loutre 1991) and, on shorter timescales, with a marked trajectory change in Northern Hemisphere solar irradiance levels: after ca. 3,000 years of general increase (to a Holocene maximum in the tree ring ${ }^{14} \mathrm{C}-$ based reconstruction of Vieira et al. 2011), there is a noted shift 


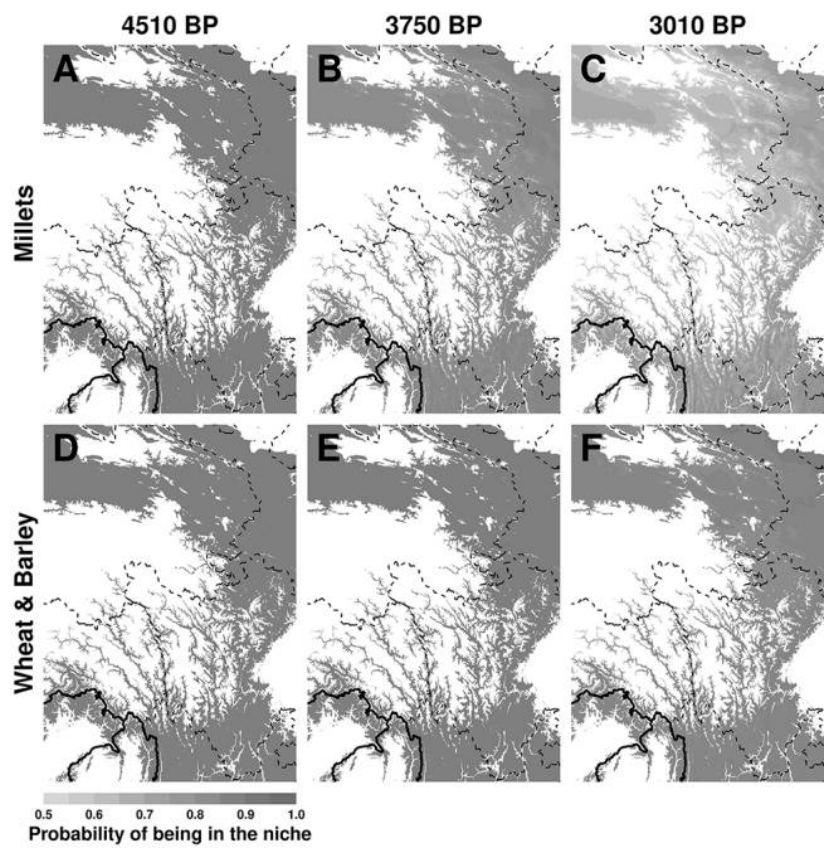

Figure 2. Thermal growing niches for millets (top) and wheat and barley (bottom) on the eastern Tibetan Plateau. Colors range from $50 \%$ probability (light gray) to $100 \%$ probability (dark gray). While growing millets $(A, B, C)$ is decreasingly viable between 4500 and $3000 \mathrm{BP}$, wheat and barley $(D, E, F)$ remain abundantly in the thermal niche. By 3000 BP, people on the eastern Tibetan Plateau had ceased cultivating millets. $A$, Millets, 4510 BP. $B$, Millets, 3750 BP. C, Millets, 3010 BP. D, Wheat and barley, 4510 BP. E, Wheat and barley, 3750 BP. F., Wheat and barley, 3010 BP. The eastern Tibetan Plateau is as outlined in figure 1. See video A1 for additional years. A color version of this figure is available online.

to decreasing values from ca. 4200-3900 BP (fig. A4). The changes observed in East Asia generally correspond with or follow the "cool poles, dry tropics" rapid climate change episode ca. 4200-3800 BP (Mayewski et al. 2004), much discussed in Old World archaeology (Meller et al. 2015).

Although wheat and barley arrive in East Asia at ca. 4500 BP (Barton and An 2014; Betts et al. 2013; Dodson et al. 2013; Flad et al 2010; Li et al. 2007; Li and Mo 2004; Zhao 2009) and are found on the NETP as early as this date, the number of finds, in terms of the overall assemblage, is small, and they appear to be minor (if not traded) crops until after 3500 BP. It is likely that farmers opted to continue to grow the more familiar millets until decreasing yields and crop failure lead to increased experimentation with wheat and barley.

The way in which humans coped with climatic cooling from 4500 to $3500 \mathrm{BP}$ appears to have differed between the NETP and the SETP (fig. 4; table A1; tables A1, A2 available in CA+ supplement A). A notable gap exists between the cessation of millet use and the arrival of wheat and barley on the SETP (fig. 4C). It is possible that the lack of availability of a suitable crop when faced with cooling temperatures could have led to an abandonment of this region. Despite cultural connections with northwest China during the initial period of occupation of the SETP, the lack of evidence for clear and continued exchange suggests that long-distance interregional exchange in the area may have been in decline in the SETP between 4500 and 3500 BP. For instance, while pottery from the Miaodigou (ca. 6000-5500 BP) and Majiayao phases (ca. 5500$4500 \mathrm{BP}$ ) appears in sites along the SETP, there is no evidence for pottery from the later Banshan (ca. 4500-4300 BP) and Machang (ca. 4300-4000 BP) cultural phases in the region (Chen 2007). Interregional exchange between the SETP and the NETP appears, however, to have reintensified once again following $4000 \mathrm{BP}$. The culture history of the region suggests that, when wheat and barley move into the SETP, they do so accompanied by a suite of new cultural characteristics that share strong similarities with cultures on the NETP, such as Kayue, Siwa, and Xindian (Luo 2012). On the NETP, on the other hand, the arrival of wheat and barley at ca. $4000 \mathrm{BP}$ appears to have allowed occupation of the area to continue without interruption. Although their predicted niche distribution is still confined to the lower river valleys of the Plateau, the probability of wheat and barley being in the niche in these areas remains high throughout history, although this decreases slightly following $3000 \mathrm{BP}$ (fig. 2).

A variety of estimates exist for the numbers of GDDs required by barley (table A2) - the lowest recorded GDDs for barley is 1,200 GDDs and is derived from a field study in Alaska (Dofing 1995), whereas studies performed in temperate areas place barley's GDD requirements at between 1,800 and 2,200 GDDs (McMaster et al. 2011). Cultivars developed in temperate areas have slower grain fill rates, making them

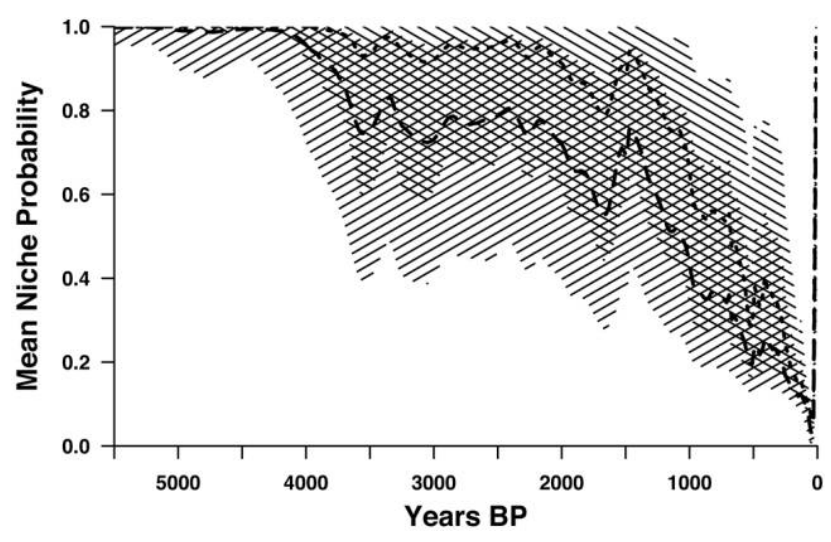

Figure 3. Mean probability of being in the thermal growing niche through time. Millets (lines upward sloping from left to right) decrease precipitously between 4000 and $3500 \mathrm{BP}$, while wheat and barley (downward sloping lines from left to right) remain largely in the niche. Dotted lines are at the mean probability for the Marcott et al. (2013) Northern Hemisphere temperature reconstruction. The upper line represents wheat and barley, and the lower line represents millets. A color version of this figure is available online. 


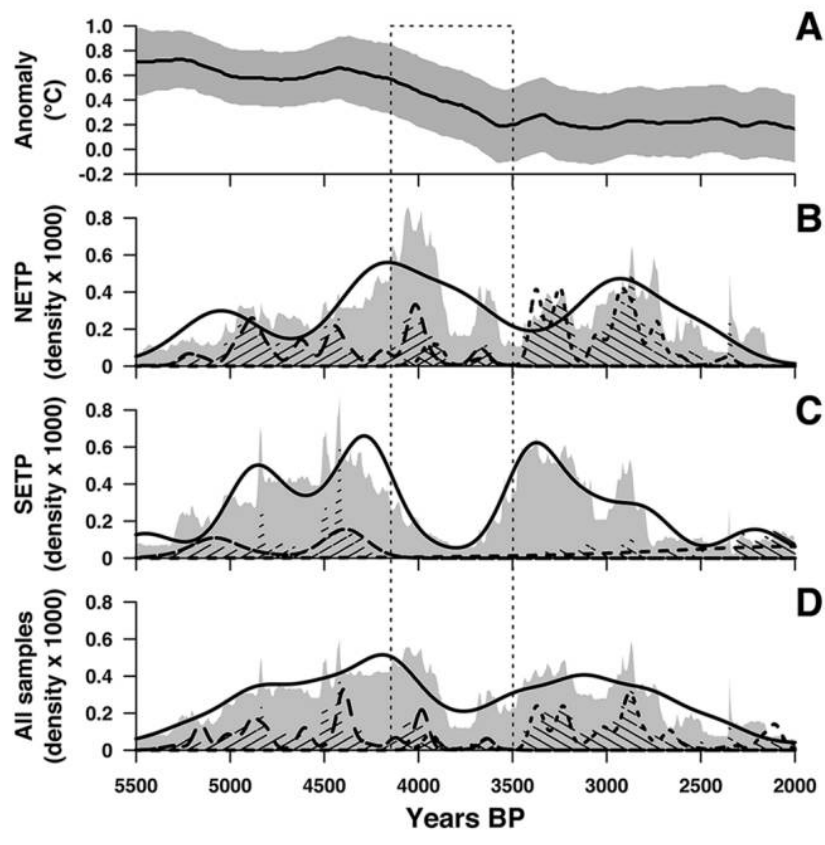

Figure 4. Radiocarbon dates on the Tibetan Plateau. A, Marcott et al. (2013) temperature reconstruction. B, Radiocarbon dates on the northeastern Tibetan Plateau (NETP). $C$, Radiocarbon dates on the southeastern Tibetan Plateau (SETP). D, Dates across the Tibetan Plateau. For $B, C$, and $D$, shaded areas in background are summed probability density distributions, while lines in the foreground represent a nonparametric phase model on ${ }^{14} \mathrm{C}$ ages via Gaussian mixture density estimation; lines upward sloping from left to right represent dates from millets; downward sloping lines from left to right represent dates from wheat and barley; and gray represents all available dates. The dotted box represents the transition period with both millet-derived and wheat- and barleyderived probability density distributions: $4,146-3,498 \mathrm{cal} \mathrm{BP}$. All radiocarbon dates have been calibrated. A color version of this figure is available online.

less adapted to cooler environments. In areas with short growing seasons, varieties have been developed that allow grain filling and flowering to take place earlier (Dofing 1995; Juskiw et al. 2001; Lopez-Castaneda and Richards 1994; table A2). Genetic evidence has revealed that barley (and wheat) domesticated in the Near East had a winter habit: these barleys were adapted to areas with relatively warm winters that could sustain plant growth (Takahashi et al. 1963, 1968; von Bothmer et al. 2003). Mutations that result in a spring phenotype appear to have taken place multiple times throughout history (Pourkheirandish and Komatsuda 2007; Takahashi et al. 1963, 1968; von Bothmer et al. 2003). One of the prerequisites for the northward expansion of barley (and of wheat) was the development of a spring phenotype (Jones et al. 2011; Lister at al. 2009). The development of this phenotype may have also been crucial for allowing its spread into high-altitude and high-latitude Eurasia (d'Alpoim Guedes 2013, 2015; d'Alpoim Guedes et al. 2013). Although the earliest forms of barley to spread into East Asia may have already developed a spring phenotype, it is unlikely that they had already developed other adaptations to high- altitude environments; there was not the requisite evolutionary pressure for these traits to be developed in the lower-elevation river valleys of the Tibetan Plateau.

Wheat and barley, on average, have an $80 \%$ probability of being in the niche until toward the start of the Little Ice Age ( 1000 BP; fig. 3 ). The extreme cooling period that represents the last 1,000 years (until the twentieth century; fig. A3) may have placed considerable adaptive pressure on local varieties of wheat and barley on the Tibetan Plateau. It is likely that it is during this period of time and not earlier that short-season cold-tolerant varieties that are present today at high altitudes and high latitudes were developed by farmers.

Although no historical records document these innovations on the Tibetan Plateau, historical records indicate that, between 1730 and $1850 \mathrm{AD}$, cooling climate may have impacted grain yields across China (Lee 2014). Additionally, strong genetic links exist between Tibetan wild barley and Tibetan naked barleys (Zeng et al. 2015): cold-acclimated genes appear to have expanded in the Tibetan hulless barley lineage. This makes it possible that, following their spread to the Tibetan Plateau, foreign barley shared genetic code with local wild barleys, and indeed, this hybridization may have enhanced cold tolerance (d'Alpoim Guedes et al. 2013). If anything, we expect that early varieties of wheat and barley introduced to the eastern Tibetan Plateau had lower GDD requirements than the varieties we report here.

Wheat and barley moved into Eastern Asia at the same time that people adopted a new, more mobile way of life and began herding pastoral animals. Previous work (Chen et al. 2015; Dong et al. 2015) takes the (mere) presence of grains at high-elevation sites as evidence for in situ agriculture. However, we argue that this assumption critically overlooks attested patterns of human interaction in high-altitude environments. Pastoralists often carry grain (or other vegetable matter) with them when driving animals to higher-elevation lands that contain rich pasture (e.g., Browman 1974; d'Alpoim Guedes 2015; d'Alpoim Guedes et al. 2015; d'Alpoim Guedes, Bocinsky, and Butler 2015). It is only through careful construction of models and evaluation of our assumptions that archaeologists will be able to understand the complexity of human patterns of mobility and interaction and the history of human innovations in subsistence on the Tibetan Plateau.

Since the year 2000, worldwide temperatures have been warmer than $82 \%$ of the Holocene average, and by 2100 , temperatures will likely exceed those known over the course of the Holocene (Marcott et al. 2013). The Tibetan Plateau is warming at a rate that is two times that of the global average ( $\mathrm{Xu}$ and Grumbine 2014; Xu et al. 2009), an alarming trend that poses great challenges for agricultural and pastoral regimes (Haynes et al. 2014; Yu et al. 2010) and puts the livelihoods of millions of smallholder farmer families at risk. Global warming is likely to extend the available niche for wheat and barley but also once again allow for cultivation of millets on the Tibetan Plateau, whereas land available for pasture is likely to decrease (Haynes et al. 2014). As temperatures increase, tra- 
ditional crops, such as millets, may once again become important staples on the Plateau.

\section{Acknowledgments}

The data and full code used to produce the figures in this manuscript are contained in a zip file, available in $\mathrm{CA}+$ supplement A. J. d'Alpoim Guedes is grateful for funding from an American Council of Learned Societies and Henry Luce Foundation Postdoctoral Fellowship in China Studies. R. K. Bocinsky received support from the National Science Foundation (BCC-1439603, DGE-1347973, and DEB-0816400). We are grateful for the comments provided by three anonymous reviewers who greatly helped improve this manuscript.

\section{References Cited}

Barton, Loukas, and Chengbang An. 2014. An evaluation of competing hypotheses for the early adoption of wheat in East Asia. Word Archaeology 46 (5):775-798.

Berger, André, and Marie-France Loutre. 1991. Insolation values for the climate of the last 10 million years. Quaternary Science Reviews 10:297-317.

Betts, Alison, Peter Weiming Jia, and John R. Dodson. 2013. The origins of wheat in China and potential pathways for its introduction: a review. Quarternary International 348(20):158-168.

Bocinsky, R. Kyle, and Timothy A. Kohler. 2014. A 2,000-year reconstruction of the rain-fed maize agricultural niche in the US Southwest. Nature Communications 5:5618. doi:10.1038/ncomms6618.

Browman, David L. 1974. Pastoral nomadism in the Andes. Current Anthropology 15:188-196.

Chen, Fahu, Guanghui Dong, Dongju Zhang, Xinyi Liu, Chengban An, Mimin Ma, Loukas Barton, et al. 2015. Agriculture facilitated permanent human occupation of the Tibetan Plateau after 3600 B.P. Science 347 (6219):248-250.

Chen, Jian. 2007. Boxi, Yingpanshan and Shawudu: the cultural development of Neolithic cultures in the Upper Min River (in Chinese). Kaogu Yu Wenwu 2007(5):65-70.

d'Alpoim Guedes, Jade. 2013. Adaptation and invention during the spread of agriculture to southwest China. PhD dissertation, Harvard University. . 2015. Rethinking the spread of agriculture to the Tibetan Plateau. The Holocene. doi:10.1177/0959683615585835.

d'Alpoim Guedes, Jade, and Ethan Butler. 2014. Modeling constraints on the spread of agriculture to Southwest China with thermal niche models. Quaternary International 349(2014):29-41.

d'Alpoim Guedes, Jade, Hongliang Lu, Anke Hein, and Amanda Schmidt. 2015. Early evidence for the use of wheat and barley as staple crops on the margins of the Tibetan Plateau. Proceedings of the National Academy of Sciences of the U S A. 112(18):5625-5630.

d'Alpoim Guedes, Jade, R. Kyle Bocinsky, and Ethan Butler. 2015. Comment on "agriculture facilitated permanent occupation of the Tibetan Plateau." Science 348(6237):872b.

d'Alpoim Guedes, Jade, Hongliang Lu, Yongxian Li, Robert N. Spengler, Xiaohong $\mathrm{Wu}$, and Mark Aldenderfer. 2013. Moving agriculture onto the Tibetan Plateau: the archaeobotanical evidence. Archaeological and Anthropological Sciences 6:255-269.

Dodson, John R., Xiaoqiang Li, Xinying Zhou, Keliang Zhao, Nan Sun, and Pia Atahan. 2013. Origin and spread of wheat in China. Quaternary Science Reviews 72(2013):108-111.

Dofing, Sean M. 1995. Phenological development-yield relationships in spring barley in a subartic environment. Canadian Journal of Plant Sciences 75(1): 93-97.

Dong, Guanghui, Dongju Zhang, Xinyi Liu, Fengwen Liu, Fahu Chen, and Martin Jones. 2015. Response to comment on "agriculture facilitated permanent occupation of the Tibetan Plateau after 3600 B.P." Science 348(6237):872c.

Dykoski, Carolyn A., R. Lawrence Edwards, Hai Cheng, Daoxian Yuan, Yanjun Cai, Meiliang Zhang, Yushi Lin, Jiaming Qing, Zhisheng An, and Justin Revenaugh. 2005. A high-resolution, absolute-dated Holocene and deglacial Asian monsoon record from Dongge Cave, China. Earth and Planetary Science Letters 233:71-86.

Flad, Rowan, Shuicheng Li, Wu Xiaohong, and Zhijun Zhao. 2010. Early wheat in China: results from new studies at Donghuishan in the Hexi Corridor. Holocene 17:555-560.

Haynes, Michelle A., King-Jau Samuel Kung, Jodi S. Brandt, Yang Yongping, and Donald M. Waller. 2014. Accelerated climate change and its potential impact on Yak herding livelihoods in the eastern Tibetan plateau. Climatic Change 123(2):147-160.

Jones, Huw, Peter Civan, James Cockram, Fiona Leigh, Lydia Smith, Martin Jones, Michael Charles, et al. 2011. Evolutionary history of barley cultivation in Europe revealed by genetic analysis of extant landraces. $\underline{B M C}$ Evolutionary Biology 11(1):320.

Juskiw, Patricia E., Yih-Wu Jame, and Len Kryzanowski. 2001. Phenological development of spring barley in a short-season growing area. Agronomy Journal 93:370-379. Kapstein, Mathew. 2006. The Tibetans. New York: Wiley-Blackwell.

Lee, Harry. 2014. Climate-induced agricultural shrinkage and overpopulation in late imperial China. Climate Research 59:229-242.

Lister, Diane L., Susan Thaw, Mim A. Bower, Huw Jones, Michael P. Charles, Glynis Jones, Lydia M. J. Smith, Christopher J. Howe, Terence A. Brown, and Martin K. Jones. 2009. Latitudinal variation in a photoperiod response gene in European barley: insight into the dynamics of agricultural spread from "historic" specimens. Journal of Archaeological Science 36(2009):1092-1098.

Li, Xiaoqiang, John Dodson, Xinying Zhou, Hongbin Zhang, and Ryo Masutomoto. 2007. Early cultivated wheat and broadening of agriculture in Neolithic China. The Holocene 17:555-560.

Li, Shuicheng, and Duowen Mo. 2004. Considering the dating of wheat at the site of Donghuishan (in Chinese). Kaogu yu Wenwu 2004(6):51-60. Lopez-Castaneda, Candido, and Richard A. Richards. 1994. Variation in temperate cereals in rainfed environments: II. Phasic development and growth. Field Crops Research 37(1):63-75.

Luo, Erhu. 2012. Culture, environment, society, groups: research on the stone-cist graves of the ethnic corridor of Southwest China. Beijing: Kexue Chubanshe.

Marcott, Shaun A., Jeremy D. Shakun, Peter U. Clark, and Alan C. Mix. 2013. A reconstruction of regional and global temperature for the past 11,300 years. Science 339(6124):1198-1201.

Mayewski, Paul A., Eelco E. Rohling, J. Curt Stager, Wibjörn Karlén, Kirk A. Maasch, L. David Meeker, Eric A. Meyerson, et al. 2004. Holocene climate variability. Quaternary Research 62:243-255.

McMaster, Gregory S., Deborah. A. Edmunds, Will W. Wilhelm, David. C. Nielsen, P. V. Vara Prasad, and James C. Ascough II. 2011. Phenology MMS: a program to simulate crop phenological responses to water stress. Computers and Electronics in Agriculture 77(1):118-125.

Meller, Harald, Helge Wolfgang Arz, Reinhard Jung, and Roberto Risch, eds. 2015. 2200 BC: a climatic breakdown as a cause for the collapse of the old world (in German). Halle, Germany: Landesamt für Denkmalpflege Sachsen-Anhalt.

Pourkheirandish, Mohammad, and Takao Komatsuda. 2007. The importance of barley genetics and domestication in a global perspective. Annals of Botany 100(5):999-1008.

Rehfeld, Kira, and Thomas Laepple. 2016. Warmer and wetter or warmer and dryer? observed versus simulated covariability of Holocene temperature and rainfall in Asia. Earth and Planetary Science Letters 436:1-9.

Takahashi, Ryuhei, Jiro Hayashi, Unji Hiura, and Shozo Yasuda. 1968. A study of cultivated barleys from Nepal, Himalaya and North India with special reference to their phylogenetic differentiation. Bericht des Ohara Instituts für Landwirtschaftliche Biologie 14:85-122.

Takahashi, Ryuhei, Jiro Hayashi, Shozo Yasuda, and Unji Hiura. 1963. Characteristics of the wild and cultivated barleys from Afghanistan and its neighboring regions. Bericht des Ohara Instituts für Landwirtschaftliche Biologie, Okayama 1:1-23.

von Bothmer, Roland, Theo van Hintum, Helmut Knüpffer, and Kazuhiro Sato. 2003. Diversity in barley: developments in plant genetics and breeding. Amsterdam, NE: Elsevier.

Vieira, Luis Eduardo A., Sami K. Solanki, Natalie A. Krovova, and Ilya Usoskin. 2011. Evolution of the solar irradiance during the Holocene. Astronomy and Astrophysics 531(A6). doi:10.1051/0004-6361/201015843.

$\mathrm{Xu}$, Jianchu, and Edward R. Grumbine. 2014. Building ecosystem resilience for climate change adaptation in the Asian highlands. WIREs Climatic Change 5:709-718.

Xu, Jianchu, Edward R. Grumbine, Arun Shrestha, Mats Eriksson, Xuefei Yang, Yun Wang, and Andrea Wilkes. 2009. The melting Himalayas: cascading effects of climate change on water, biodiversity and livelihoods. Conservation Biology 23:520-530. 
Yu, Haiyang, Eieke Luedeling, and Jianchu Xu. 2010. Winter and spring warming result in delayed spring phenology on the Tibetan Plateau. Proceedings of the National Academy of Sciences of the U S A 107:22151-22156.

Zeng, Xingquan, Hai Long, Zhuo Wang, Shancen Zhao, Yawei Tang, Zhiyong Huang, Yulin Wanga, et al. 2015. The draft genome of Tibetan hulles barley reveals adaptive patterns to the high stressful Tibetan Plateau. Proceedings of the National Academy of Sciences of the US A 112 (4):10951100.

Zhao, Zhijun. 2009. Eastward spread of wheat into China: new data and new issues. Chinese Archaeology 9(1):1-9.

This content downloaded from 134.121.161.015 on February 04, 2017 10:01:52 AM 